\title{
Onset of swell-induced surface instability of hydrogel layers with depth-wise graded material properties
}

\author{
Zhigen $\mathrm{Wu}^{\mathrm{a}, *}$, Nikolaos Bouklas ${ }^{\mathrm{b}}$, Yihua Liu ${ }^{\text {a }}$, Rui Huang ${ }^{\mathrm{b}, *}$ \\ ${ }^{a}$ School of Civil Engineering, Hefei University of Technology, Hefei, Anhui 230009, China \\ ${ }^{\mathrm{b}}$ Department of Aerospace Engineering and Engineering Mechanics, University of Texas, \\ Austin, TX 78712, USA
}

\begin{abstract}
A rich variety of instability patterns have been observed on the surface of hydrogel layers with depth-wise variation in material properties. In this paper, a state space method and a finite difference method are developed to predict the critical condition for onset of surface instability for hydrogel layers with continuously graded material properties in the thickness direction. Both methods are benchmarked by comparing to analytical solutions for homogeneous hydrogel layers and hydrogel bilayers. While the finite difference method often requires a large number of nodes to achieve convergence, the state space method requires relatively fewer sub-layers for continuously graded layers. The results for linearly and exponentially graded hydrogel layers show that the critical swelling ratio and corresponding critical wavelength both depend on the gradient profile of the crosslink density. The present study may provide theoretical guidance for analyzing and designing surface instability and surface patterns in hydrogel layers.
\end{abstract}

Keywords: Hydrogel; Surface instability; State space method; Finite difference method

\footnotetext{
${ }^{*}$ Corresponding authors.

E-mail addresses: zhigenwu@hfut.edu.cn (Z.Wu), ruihuang@mail.utexas.edu (R. Huang).
} 


\section{Introduction}

A rich variety of swell-induced surface instability patterns have been observed with polymer gels (Tanaka et al., 1987, 1992; Sultan and Boudaoud, 2008; Trujillo et al. 2008; Guvendiren et al., 2009). This phenomenon has been exploited for a range of applications, including sensors (Schaffer et al., 2000; Stafford et al., 2004), microfluidic devices (Beebe et al., 2000; Sugiura et al., 2007), micro-optics (Harrison et al., 2004), and active surfaces (Tokarev and Minko, 2009). Theoretical studies on the swell-induced surface instability of hydrogels are important for two reasons: (1) they provide fundamental understanding on the mechanisms for the formation of surface patterns; (2) they offer analytical methods for the design and exploitation of potential applications.

For a homogeneous hydrogel layer on a rigid substrate, Kang and Huang (2010a) predicted that the critical swelling ratio for onset of surface wrinkling instability varies from about 2.5 to 3.4 , depending on the material parameters (both the polymer network and the solvent). By considering the effect of surface tension, a characteristic wrinkle wavelength that scales almost linearly with the thickness of the hydrogel layer was predicted (Kang and Huang, 2010b). On the other hand, it was noted that the surface wrinkling of a homogeneous hydrogel layer is highly unstable and may be preceded by formation of surface creases. Based on an energetic consideration and numerical calculations, Weiss et al. (2013) predicted that the critical swelling ratio for the onset of surface creasing is considerably lower than that for wrinkling.

Recently, a series of experiments by Guvendiren et al. (2009, 2010a, 2010b) have observed a variety of surface patterns (including creases and wrinkles) by using hydrogels with depth-wise crosslink gradients. In their experiments, the critical swelling ratios were reported to be 1.12 for wrinkling and in the range of 1.3 2.0 for creasing, both considerably lower than the counterparts for a homogeneous layer. Wu et al. (2013) presented a linear perturbation analysis for hydrogel layers with material properties varying in the thickness direction and obtained analytical solutions to the eigenvalue problem for hydrogel bilayers with different combinations of material properties. In general, however, the material properties may vary continuously in the thickness direction, for which numerical methods are needed to solve the eigenvalue problem. In this paper, we present a state space method and a finite difference method to predict the critical condition for onset of swell-induced surface instability for hydrogel layers with continuously graded material properties in the thickness direction. 
We note that Cao et al. (2012) have considered an elastic system consisting of a hard surface layer on an elastically graded soft substrate and presented analytical solutions for the soft substrate with the elastic modulus varying as a power function and an exponential function. A similar eigenvalue problem was investigated by a finite element method for an elastic half space with graded material properties (Lee et al., 2008). Diab and Kim (2014) presented first and second-order bifurcation analysis for a neo-Hookean elastic half space with a graded-stiffness, supplemented by finite-element analysis, to predict various instability-order-dependent bifurcations, from stable wrinkling of the first order to creasing of the infinite-order cascade instability. Recently, a state space method was developed for surface instability of the elastic layers with arbitrarily graded material properties (Wu et al., 2014). The state space method was found to be computationally effective for the elastic layers in comparison with the finite element method. In the present study, we extend the state space method for surface instability of hydrogel layers where elastic deformation is coupled with solvent-induced swelling.

The remainder of this paper is organized as follows. Section 2 briefly reviews the linear perturbation analysis for hydrogel layers with material properties varying in the thickness direction. The state space method and the finite difference method are presented in Section 3 and Section 4, respectively. The results are discussed in Section 5, in comparison with the analytical solutions for homogeneous hydrogel layers and hydrogel bilayers, followed by linearly and exponentially graded hydrogel layers. Section 6 concludes the present study with a short summary. 


\section{Linear perturbation analysis}

This section briefly reviews constrained swelling and linear perturbation analysis for a depth-wise graded hydrogel layer attached to a rigid substrate. More details can be found in Wu et al. (2013).

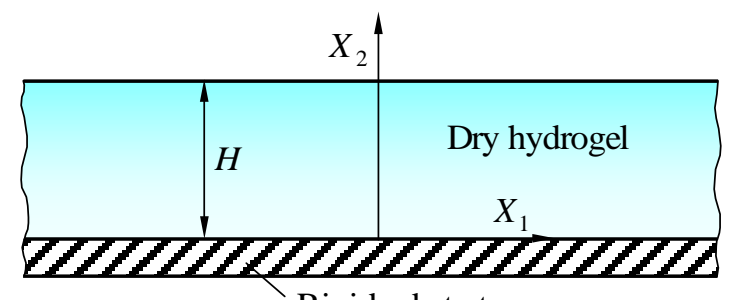

Rigid substrate

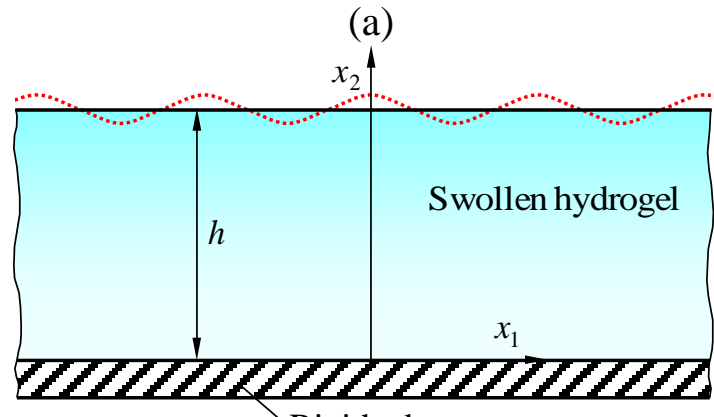

Rigid substrate

(b)

Fig. 1. Schematics of a depth-wise graded hydrogel layer on a rigid substrate: (a) the dry state; (b) a transversely homogeneous swollen state (the dotted line denotes a perturbation to the surface of the swollen state).

\subsection{Transversely homogeneous, constrained swelling}

Consider a depth-wise graded hydrogel layer attached to a rigid substrate as shown in Fig. 1a. Set a Cartesian coordinate system in the dry state such that $X_{2}=0$ at the hydrogel/substrate interface. The thickness of the hydrogel layer is $H$ and the other two dimensions are assumed to be infinite. The material properties of the hydrogel may vary with respect to $X_{2}$, i.e., $N=N\left(X_{2}\right)$ and $\chi=\chi\left(X_{2}\right)$, where $N$ is the effective number of polymer chains per unit volume of the polymer network in the dry state and $\chi$ is the Flory parameter for the interaction between solvent molecules and the polymer.

Immersed in a solvent, the graded hydrogel layer swells in the thickness direction but constrained in the in-plane directions (assuming perfect bonding at the interface), thus a transversely homogeneous swelling occurs (Fig. 1b). The local swelling ratio of the graded hydrogel layer $\lambda_{h}\left(X_{2}\right)$ is equivalent to that for a transversely constrained, homogeneous hydrogel layer with the material properties $N=N\left(X_{2}\right)$ and $\chi=\chi\left(X_{2}\right)$. The nominal stress 
in the hydrogel layer is obtained as a function of the swelling ratio and the chemical potential of solvent. By setting the normal stress on the surface to be the external solvent pressure as the boundary condition, we obtain a nonlinear equation for the local swelling ratio (Wu et al. 2013):

$$
\log \left(1-\frac{1}{\lambda_{h}}\right)+\frac{1}{\lambda_{h}}+\frac{\chi}{\lambda_{h}^{2}}+N \Omega\left(\lambda_{h}-\frac{1}{\lambda_{h}}\right)=\frac{\mu-p \Omega}{k T},
$$

where $\mu$ is the chemical potential of the solvent, $p$ is the external solvent pressure, $\Omega$ is the volume per solvent molecule, $k$ is the Boltzmann constant, and $T$ is the absolute temperature. In the equilibrium state, the chemical potential of the solvent is a constant in the hydrogel and equal to that of the external solvent, $\hat{\mu}$. For given $\mu=\hat{\mu}$ and $p$, the local swelling ratio $\lambda_{h}$ depends on the graded material properties, $N\left(X_{2}\right)$ and $\chi\left(X_{2}\right)$. At equilibrium, the total thickness of the graded hydrogel layer can be obtained by integrating the local swelling ratio, i.e., $h=\int_{0}^{H} \lambda_{h} \mathrm{~d} X_{2}$, and the average swelling ratio is then $\lambda=h / H$.

The transversely constrained swelling leads to in-plane compressive stresses, $s_{11}=s_{33}=-N k T\left[\lambda_{h}^{2}-1\right]-p \lambda_{h}$, and $s_{22}=-p$, where $s_{i j}$ denotes the nominal stress in the hydrogel. The in-plane compressive stresses may be partly relaxed by buckling. As a result, the transversely homogeneous swollen state may become unstable, giving rise to surface wrinkles or creases.

\subsection{Linear perturbation analysis}

To analyze stability of the constrained swollen state of a hydrogel layer, a small perturbation is assumed with displacements from the swollen state in the general form:

$$
u_{1}=u_{1}\left(x_{1}, x_{2}\right), \quad u_{2}=u_{2}\left(x_{1}, x_{2}\right) .
$$

With reference to the dry state, the deformation gradient tensor after the perturbation becomes

$$
\tilde{\mathbf{F}}=\left[\begin{array}{ccc}
1+\frac{\partial u_{1}}{\partial x_{1}} & \lambda_{h} \frac{\partial u_{1}}{\partial x_{2}} & 0 \\
\frac{\partial u_{2}}{\partial x_{1}} & \lambda_{h}\left(1+\frac{\partial u_{2}}{\partial x_{2}}\right) & 0 \\
0 & 0 & 1
\end{array}\right]
$$

The corresponding nominal stress components can be obtained from differentiation of the free energy density function of the hydrogel as follows (Wu et al. 2013): 


$$
\begin{aligned}
& s_{11} \approx N k T\left[\left(1+\lambda_{h} \xi_{h}\right) \frac{\partial u_{1}}{\partial x_{1}}+\lambda_{h}\left(\xi_{h}-\lambda_{h}\right) \frac{\partial u_{2}}{\partial x_{2}}-\lambda_{h}^{2}+1\right]-p \lambda_{h}\left(1+\frac{\partial u_{2}}{\partial x_{2}}\right), \\
& s_{12} \approx N k T \lambda_{h}\left(\frac{\partial u_{1}}{\partial x_{2}}+\frac{\partial u_{2}}{\partial x_{1}}\right)+p \frac{\partial u_{2}}{\partial x_{1}}, \\
& s_{21} \approx N k T\left(\lambda_{h}^{2} \frac{\partial u_{1}}{\partial x_{2}}+\frac{\partial u_{2}}{\partial x_{1}}\right)+p \lambda_{h} \frac{\partial u_{1}}{\partial x_{2}}, \\
& s_{22} \approx N k T\left[\left(\xi_{h}-\lambda_{h}\right) \frac{\partial u_{1}}{\partial x_{1}}+\left(\xi_{h}+\lambda_{h}\right) \frac{\partial u_{2}}{\partial x_{2}}\right]-p\left(1+\frac{\partial u_{1}}{\partial x_{1}}\right), \\
& s_{33} \approx-N k T\left[\lambda_{h}^{2}(1+\varepsilon)-\lambda_{h} \xi_{h} \varepsilon-1\right]-p \lambda_{h}(1+\varepsilon),
\end{aligned}
$$

where $\xi_{h}=\frac{1}{\lambda_{h}}+\frac{1}{N \Omega}\left(\frac{1}{\lambda_{h}-1}-\frac{1}{\lambda_{h}}-\frac{2 \chi}{\lambda_{h}^{2}}\right)$, and $s_{13}=s_{31}=s_{23}=s_{32}=0$.

In the absence of body force, the mechanical equilibrium requires that the nominal stress components must satisfy

$$
\begin{aligned}
& \frac{\partial s_{11}}{\partial x_{1}}+\lambda_{h} \frac{\partial s_{12}}{\partial x_{2}}=0, \\
& \frac{\partial s_{21}}{\partial x_{1}}+\lambda_{h} \frac{\partial s_{22}}{\partial x_{2}}=0 .
\end{aligned}
$$

Substituting Eqs. (4)-(7) into Eqs. (9) and (10), we obtain two coupled linear differential equations in terms of the perturbation displacements:

$$
\begin{aligned}
& \left(1+\lambda_{h} \xi_{h}\right) \frac{\partial^{2} u_{1}}{\partial x_{1}^{2}}+\lambda_{h}^{2} \frac{\partial^{2} u_{1}}{\partial x_{2}^{2}}+\lambda_{h} \xi_{h} \frac{\partial^{2} u_{2}}{\partial x_{1} \partial x_{2}}+f_{1}\left(x_{2}\right)\left(\frac{\partial u_{1}}{\partial x_{2}}+\frac{\partial u_{2}}{\partial x_{1}}\right)=0, \\
& \frac{\partial^{2} u_{2}}{\partial x_{1}^{2}}+\lambda_{h}\left(\xi_{h}+\lambda_{h}\right) \frac{\partial^{2} u_{2}}{\partial x_{2}^{2}}+\lambda_{h} \xi_{h} \frac{\partial^{2} u_{1}}{\partial x_{1} \partial x_{2}}+f_{2}\left(x_{2}\right) \frac{\partial u_{1}}{\partial x_{1}}+f_{3}\left(x_{2}\right) \frac{\partial u_{2}}{\partial x_{2}}=0,
\end{aligned}
$$

where $f_{1}\left(x_{2}\right)=\frac{\lambda_{h}}{N \Omega} \frac{\mathrm{d}}{\mathrm{d} x_{2}}\left(\lambda_{h} N \Omega\right), f_{2}\left(x_{2}\right)=\frac{\lambda_{h}}{N \Omega} \frac{\mathrm{d}}{\mathrm{d} x_{2}}\left[N \Omega\left(\xi_{h}-\lambda_{h}\right)\right]$, and

$$
f_{3}\left(x_{2}\right)=\frac{\lambda_{h}}{N \Omega} \frac{\mathrm{d}}{\mathrm{d} x_{2}}\left[N \Omega\left(\xi_{h}+\lambda_{h}\right)\right]
$$

Take the perturbation displacements to be periodic in the $x_{1}$-direction, namely

$$
u_{1}=U_{1}\left(x_{2}\right) \sin \omega x_{1} \text { and } u_{2}=U_{2}\left(x_{2}\right) \cos \omega x_{1},
$$

where $\omega$ is the wave number. Substitution of Eq. (13) into Eqs. (11) and (12) yields

$$
\lambda_{h}^{2} U_{1}^{\prime \prime}+f_{1} U_{1}^{\prime}-\omega^{2}\left(1+\lambda_{h} \xi_{h}\right) U_{1}-\omega \lambda_{h} \xi_{h} U_{2}^{\prime}-\omega f_{1} U_{2}=0,
$$




$$
\omega \lambda_{h} \xi_{h} U_{1}^{\prime}+\omega f_{2} U_{1}+\lambda_{h}\left(\xi_{h}+\lambda_{h}\right) U_{2}^{\prime \prime}+f_{3} U_{2}^{\prime}-\omega^{2} U_{2}=0
$$

where the single and double primes denote the first and second derivatives of $U_{1}\left(x_{2}\right)$ and $U_{2}\left(x_{2}\right)$.

The bottom surface of the hydrogel layer is attached to the rigid substrate with zero displacements, namely

$$
U_{1}=U_{2}=0, \quad \text { at } \quad x_{2}=0 .
$$

The top surface of the hydrogel layer is subjected to a pressure from the external solvent. To the first order of the perturbation, the nominal traction at the surface is

$$
s_{12}=p \frac{\partial u_{2}}{\partial x_{1}} \quad \text { and } \quad s_{22}=-p\left(1+\frac{\partial u_{1}}{\partial x_{1}}\right), \quad \text { at } \quad x_{2}=h .
$$

Thus, by Eqs. (5), (7), and (13) the top surface satisfies the boundary condition:

$$
U_{1}^{\prime}-\omega U_{2}=0 \text { and }\left(\xi_{h}-\lambda_{h}\right) \omega U_{1}+\left(\xi_{h}+\lambda_{h}\right) U_{2}^{\prime}=0, \text { at } x_{2}=h .
$$

The equilibrium equations (14) and (15) along with the boundary conditions (16) and (18) constitute an eigenvalue problem for the constrained swollen state. If there exists a nontrivial solution for any wave number $\omega$, the transversely homogeneous swollen state of the hydrogel layer becomes unstable. Constrained at the bottom by the rigid substrate (assuming no debonding), surface instability modes (wrinkles or creases) are expected. Thus, by solving the eigenvalue problem, the critical condition is predicted for the onset of swell-induced surface instability. However, for an arbitrarily graded hydrogel layer with $N\left(X_{2}\right)$ and $\chi\left(X_{2}\right)$, the eigenvalue problem in general cannot be solved analytically. In Sections 3 and 4, we develop a state space method and a finite difference method to solve the eigenvalue problem for hydrogel layers with depth-wise graded material properties.

\section{A state space method}

The state space method is commonly used in dynamic systems to analyze multiple inputs and outputs related by differential equations (also known as the "time-domain approach") (Elgerd, 1967; Derusso et al., 1998). Recently, the state space method has been adopted as an effective analytical approach for surface instability analysis in elastic layers (Wu et al., 2014; Toh et al., 2015). In this section, we extend this method to determine the critical condition for swell-induced surface instability of hydrogel layers.

First consider a homogeneous hydrogel layer. Both material properties $N$ and $\chi$ are independent of $X_{2}$, and the quantities $\lambda_{h}$ and $\xi_{h}$ are constants. From Eqs. (5) and (7), we 
have

$$
\begin{aligned}
& \frac{\partial u_{1}}{\partial x_{2}}=-\left(1+\frac{p}{N k T \lambda_{h}}\right) \frac{\partial u_{2}}{\partial x_{1}}+\frac{s_{12}}{N k T \lambda_{h}}, \\
& \frac{\partial u_{2}}{\partial x_{2}}=-\frac{N k T\left(\xi_{h}-\lambda_{h}\right)-p}{N k T\left(\xi_{h}+\lambda_{h}\right)} \frac{\partial u_{1}}{\partial x_{1}}+\frac{s_{22}+p}{N k T\left(\xi_{h}+\lambda_{h}\right)} .
\end{aligned}
$$

Differentiation of Eqs. (4) and (6) with respect to $x_{1}$ yields

$$
\begin{aligned}
& \frac{\partial s_{11}}{\partial x_{1}}=N k T\left[\left(1+\lambda_{h} \xi_{h}\right) \frac{\partial^{2} u_{1}}{\partial x_{1}^{2}}+\lambda_{h}\left(\xi_{h}-\lambda_{h}\right) \frac{\partial}{\partial x_{1}}\left(\frac{\partial u_{2}}{\partial x_{2}}\right)\right]-p \lambda_{h} \frac{\partial}{\partial x_{1}}\left(\frac{\partial u_{2}}{\partial x_{2}}\right), \\
& \frac{\partial s_{21}}{\partial x_{1}}=N k T\left[\lambda_{h}^{2} \frac{\partial}{\partial x_{1}}\left(\frac{\partial u_{1}}{\partial x_{2}}\right)+\frac{\partial^{2} u_{2}}{\partial x_{1}^{2}}\right]+p \lambda_{h} \frac{\partial}{\partial x_{1}}\left(\frac{\partial u_{1}}{\partial x_{2}}\right) .
\end{aligned}
$$

Substituting Eqs. (19) and (20) into Eqs. (21) and (22) and then into Eqs. (9) and (10), we obtain that

$$
\begin{aligned}
& \frac{\partial s_{12}}{\partial x_{2}}=\left\{\frac{\left[N k T\left(\xi_{h}-\lambda_{h}\right)-p\right]^{2}}{N k T\left(\xi_{h}+\lambda_{h}\right)}-N k T\left(\xi_{h}+\frac{1}{\lambda_{h}}\right)\right\} \frac{\partial^{2} u_{1}}{\partial x_{1}^{2}}-\frac{N k T\left(\xi_{h}-\lambda_{h}\right)-p}{N k T\left(\xi_{h}+\lambda_{h}\right)} \frac{\partial s_{22}}{\partial x_{1}}, \\
& \frac{\partial s_{22}}{\partial x_{2}}=\frac{N k T}{\lambda_{h}}\left[\left(\lambda_{h}+\frac{p}{N k T}\right)^{2}-1\right] \frac{\partial^{2} u_{2}}{\partial x_{1}^{2}}-\left(1+\frac{p}{N k T \lambda_{h}}\right) \frac{\partial s_{12}}{\partial x_{1}} .
\end{aligned}
$$

Based on the assumption of the perturbation displacements in Eq. (13), the nominal stresses $s_{22}$ and $s_{12}$ may be written as

$$
s_{22}=S_{22}\left(x_{2}\right) \cos \omega x_{1}-p, \quad s_{12}=S_{12}\left(x_{2}\right) \sin \omega x_{1} .
$$

Inserting Eqs. (13) and (25) into Eqs. (19), (20), (23), and (24), a set of differential equations are obtained in a matrix form as

$$
\frac{\mathrm{d}}{\mathrm{d} x_{2}}\left\{\begin{array}{l}
U_{1}\left(x_{2}\right) \\
S_{22}\left(x_{2}\right) \\
U_{2}\left(x_{2}\right) \\
S_{12}\left(x_{2}\right)
\end{array}\right\}=\mathbf{A}\left\{\begin{array}{l}
U_{1}\left(x_{2}\right) \\
S_{22}\left(x_{2}\right) \\
U_{2}\left(x_{2}\right) \\
S_{12}\left(x_{2}\right)
\end{array}\right\},
$$

where $\mathbf{A}$ is a four-by-four matrix with the following nonzero elements:

$$
\begin{aligned}
& A_{13}=-A_{24}=\left(\frac{p}{N k T \lambda_{h}}+1\right) \omega, A_{14}=\frac{1}{N k T \lambda_{h}}, A_{23}=\left[\frac{N k T}{\lambda_{h}}-\frac{\left(N k T \lambda_{h}+p\right)^{2}}{N k T \lambda_{h}}\right] \omega^{2}, \\
& A_{31}=-A_{42}=-\frac{N k T\left(\xi_{h}-\lambda_{h}\right)-p}{N k T\left(\xi_{h}+\lambda_{h}\right)} \omega, A_{32}=\frac{1}{N k T\left(\xi_{h}+\lambda_{h}\right)}, \\
& A_{41}=\left\{N k T\left(\xi_{h}+\frac{1}{\lambda_{h}}\right)-\frac{\left[N k T\left(\xi_{h}-\lambda_{h}\right)-p\right]^{2}}{N k T\left(\xi_{h}+\lambda_{h}\right)}\right\} \omega^{2} .
\end{aligned}
$$


Equation (26) is a linearized state equation for a homogeneous hydrogel layer, which is a set of first-order, homogeneous, and ordinary differential equations in terms of the perturbation displacements and associated tractions. By integrating the differential equations, the state vector, [ $\left.U_{1} S_{22} U_{2} S_{12}\right]^{\mathrm{T}}$, can be determined as

$$
\left\{\begin{array}{l}
U_{1}\left(x_{2}\right) \\
S_{22}\left(x_{2}\right) \\
U_{2}\left(x_{2}\right) \\
S_{12}\left(x_{2}\right)
\end{array}\right\}=\mathbf{D}\left(x_{2}\right)\left\{\begin{array}{l}
U_{1}(0) \\
S_{22}(0) \\
U_{2}(0) \\
S_{12}(0)
\end{array}\right\},
$$

where the matrix $\mathbf{D}\left(x_{2}\right)=\mathrm{e}^{\mathbf{A} x_{2}}$ can be calculated directly by the matrix exponential operation (e.g., expm in Matlab).

Inserting Eqs (13) and (25) into Eq. (17), the boundary conditions at the upper surface $\left(x_{2}=h\right)$ become

$$
S_{22}(h)=-p \omega U_{1}(h) \text { and } S_{12}(h)=-p \omega U_{2}(h) .
$$

Applying the boundary conditions (16) and (28) to Eq. (27), we have

$$
\left\{\begin{array}{c}
U_{1}(h) \\
-p \omega U_{1}(h) \\
U_{2}(h) \\
-p \omega U_{2}(h)
\end{array}\right\}=\mathbf{D}(h)\left\{\begin{array}{c}
0 \\
S_{22}(0) \\
0 \\
S_{12}(0)
\end{array}\right\},
$$

which leads to an eigenvalue problem in terms of the tractions at the lower surface of the hydrogel layer:

$$
\begin{aligned}
& \left(D_{22}+p \omega D_{12}\right) S_{22}(0)+\left(D_{24}+p \omega D_{14}\right) S_{12}(0)=0 \\
& \left(D_{42}+p \omega D_{32}\right) S_{22}(0)+\left(D_{44}+p \omega D_{34}\right) S_{12}(0)=0 .
\end{aligned}
$$

To have a nontrivial solution, the determinant of the coefficient matrix in the coupled equations (30) and (31) must vanish, which can be expressed explicitly as

$$
\begin{aligned}
& \left(\lambda_{h}-\beta\right)\left[4 \lambda_{h}^{3} \beta-\left(\lambda_{h}^{2}+1\right)^{2}\right]\left[\mathrm{e}^{\left(1+\lambda_{h} \beta\right) \omega H}+\mathrm{e}^{-\left(1+\lambda_{h} \beta\right) \omega H}\right] \\
& +\left(\lambda_{h}+\beta\right)\left[4 \lambda_{h}^{3} \beta+\left(\lambda_{h}^{2}+1\right)^{2}\right]\left[\mathrm{e}^{\left(1-\lambda_{h} \beta\right) \omega H}+\mathrm{e}^{-\left(1-\lambda_{h} \beta\right) \omega H}\right] \\
& -16 \lambda_{h}^{2} \beta\left(\lambda_{h}^{2}+1\right)=0,
\end{aligned}
$$

where $\beta=\sqrt{\left(1+\lambda_{h} \xi_{h}\right) /\left(\lambda_{h}^{2}+\lambda_{h} \xi_{h}\right)}$. For each dimensionless wave number $\omega H$, Eq. (32) predicts a critical swelling ratio $\lambda_{\mathrm{c}}$, which depends on the two material properties $N \Omega$ and $\chi$. The corresponding chemical potential $\mu_{\mathrm{c}}$ is then obtained from Eq. (1) for onset of surface instability of a homogeneous hydrogel layer. Theoretically, there exists a short-wavelength limit for a homogeneous hydrogel layer with $\omega H \rightarrow \infty$, for which Eq. (32) 
becomes

$$
\left(\lambda_{h}-\beta\right)\left[4 \lambda_{h}^{3} \beta-\left(\lambda_{h}^{2}+1\right)^{2}\right]=0 .
$$

Equations (32) and (33) are consistent with the previous results obtained by Kang and Huang (2010a) using a different method.

Next consider a hydrogel layer with material properties varying in the thickness direction such as $N\left(X_{2}\right)$ and $\chi\left(X_{2}\right)$. The functions $N\left(X_{2}\right)$ and $\chi\left(X_{2}\right)$ may be continuous or discontinuous (such as piecewise constant functions for hydrogel bilayers or multi-layers). In either case, we would divide the layer into a number of homogeneous sub-layers (Fig. 2). In the case of a continuously graded hydrogel layer, the functions $N\left(X_{2}\right)$ and $\chi\left(X_{2}\right)$ are discretized into piecewise constant functions with $n$ sub-layers. When $n$ approaches infinity and the thickness of each sub-layer approaches zero, the discretization would eventually converge towards the continuous functions. During the calculation, this graded hydrogel layer can be analyzed approximately by the discretized sub-layers with a finite but sufficiently large $n$. Therefore, regardless of continuous or discontinuous variation in material properties, surface instability of the hydrogel layer can be analyzed by using the state equation (26) for each sub-layer, along with the continuity conditions at the interfaces between adjacent sub-layers and the boundary conditions at the top and bottom surfaces.

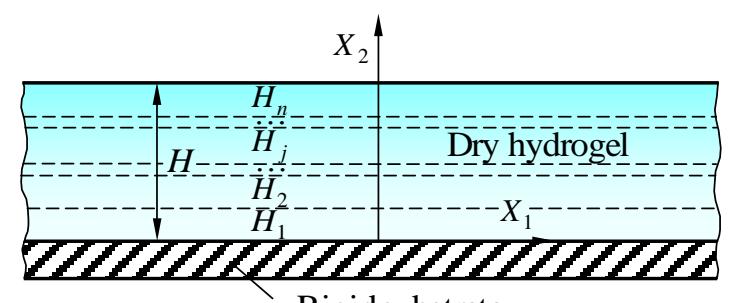

Rigid substrate

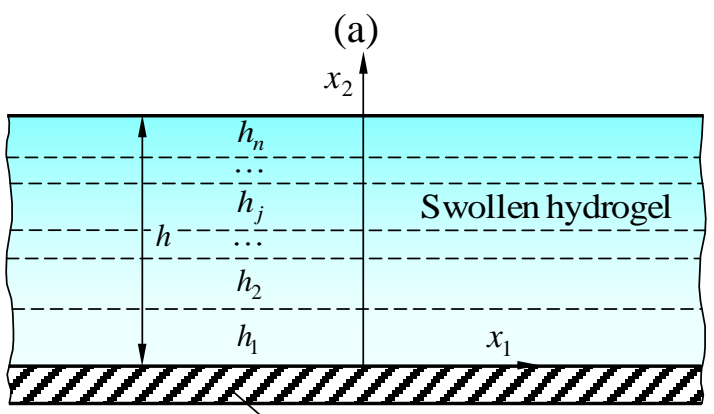

Rigid substrate

(b)

Fig. 2. A graded hydrogel layer divided into $n$ sub-layers: (a) the dry state; (b) a transversely homogeneous swollen state.

As shown in Fig. 2a, for the $j$ th sub-layer with thickness $H_{j}$, the material properties are 
approximately taken as

$$
N_{j}=\left[N\left(Y_{j-1}\right)+N\left(Y_{j}\right)\right] / 2 \text { and } \chi_{j}=\left[\chi\left(Y_{j-1}\right)+\chi\left(Y_{j}\right)\right] / 2,
$$

where $Y_{j}=H_{1}+H_{2}+\cdots+H_{j}$ and $Y_{0}=0$. In the swollen state, the thickness of the $j$ th sub-layer becomes $h_{j}$ (Fig. 2b), with a swelling ratio, $\lambda_{j}=h_{j} / H_{j}$. From the state equation (26) for each homogeneous sub-layer, the state vector at the interface $x_{2}=y_{j}$ is related to that at $x_{2}=y_{j-1}$ as

$$
\mathbf{R}\left(y_{j}\right)=\mathbf{D}_{j}\left(h_{j}\right) \mathbf{R}\left(y_{j-1}\right),
$$

where $\quad y_{j}=h_{1}+h_{2}+\cdots+h_{j} \quad, \quad y_{0}=0 \quad, \quad \mathbf{R}\left(y_{j}\right)=\left[U_{1}\left(y_{j}\right) S_{22}\left(y_{j}\right) U_{2}\left(y_{j}\right) S_{12}\left(y_{j}\right)\right]^{\mathrm{T}} \quad$, $\mathbf{D}_{j}\left(h_{j}\right)=\exp \left(\mathbf{A}_{j} h_{j}\right)$, and the matrix $\mathbf{A}_{j}$ is given by Eq. (26) for each sub-layer.

Noting the continuity of the state vector (displacements and tractions) across all interfaces between the adjacent sub-layers, we obtain the following relation:

$$
\mathbf{R}\left(y_{n}\right)=\mathbf{K R}(0),
$$

where $\mathbf{K}=\prod_{j=n}^{1} \mathbf{D}_{j}\left(h_{j}\right)$, with $\mathbf{R}(0)$ and $\mathbf{R}\left(y_{n}\right)$ being the state vectors at the bottom surface $\left(x_{2}=0\right)$ and the top surface $\left(x_{2}=y_{n}=h\right)$, respectively.

Applying the boundary conditions (16) and (28), we obtain

$$
\left\{\begin{array}{c}
U_{1}\left(y_{n}\right) \\
-p \omega U_{1}\left(y_{n}\right) \\
U_{2}\left(y_{n}\right) \\
-p \omega U_{2}\left(y_{n}\right)
\end{array}\right\}=\mathbf{K}\left\{\begin{array}{c}
0 \\
S_{22}(0) \\
0 \\
S_{12}(0)
\end{array}\right\},
$$

which leads to an eigenvalue problem in terms of the tractions at the lower surface of the hydrogel layer:

$$
\begin{aligned}
& \left(K_{22}+p \omega K_{12}\right) S_{22}(0)+\left(K_{24}+p \omega K_{14}\right) S_{12}(0)=0, \\
& \left(K_{42}+p \omega K_{32}\right) S_{22}(0)+\left(K_{44}+p \omega K_{34}\right) S_{12}(0)=0 .
\end{aligned}
$$

Similar to the case of a homogeneous hydrogel layer, the critical condition for onset of surface instability of the laminated hydrogel layer is determined by having a non-trivial solution in Eqs. (38) and (39), which requires that

$$
\left|\begin{array}{ll}
K_{22}+p \omega K_{12} & K_{24}+p \omega K_{14} \\
K_{42}+p \omega K_{32} & K_{44}+p \omega K_{34}
\end{array}\right|=0 .
$$

Equation (40) presents an implicit relation between the critical chemical potential $\mu_{\mathrm{c}}$ and the wave number $\omega$, which is associated with the discretized material properties $\left(N_{j} \Omega, \chi_{j}\right)$ and 
thickness $\left(H_{j}\right)$ of all sub-layers. By using a dimensionless wave number $\omega H$ and the normalized thickness of each sub-layer $H_{j} / H$, the critical condition can be written as

$$
\mu_{\mathrm{c}}=f_{n}\left(\omega H, N_{j} \Omega, \chi_{j}, H_{j} / H ; j=1,2, \cdots, n\right) .
$$

For each dimensionless wave number $\omega H$, we solve Eq. (40) to find the critical chemical potential $\mu_{\mathrm{c}}$. The corresponding swelling ratio of each sub-layer under the critical condition is then computed from Eq. (1), which varies with the material properties of the sub-layer. Subsequently, the critical swelling ratio for the multilayered hydrogel is obtained approximately as:

$$
\lambda_{\mathrm{c}}=\frac{h}{H}=\frac{1}{H} \sum_{j=1}^{n} \lambda_{j}\left(\mu_{\mathrm{c}}\right) H_{j} .
$$

\section{A finite difference method}

Alternatively, the general eigenvalue problem can be solved by a finite difference method to determine the critical condition for surface instability in graded hydrogel layers. Since the material properties ( $N$ and $\chi$ ) are usually provided as functions of the coordinate $X_{2}$ referring to the dry state, it is convenient to implement a uniform discretization in the dry state. However, the equilibrium equations (14) and (15) as well as the boundary condition (18) are differential equations with respect to the coordinate $x_{2}$ in the swollen state. For the finite difference method, we re-write the equilibrium equations and the boundary condition in the reference coordinate and normalize the coordinate by the dry-state thickness of the hydrogel layer, $H$. By the chain rule, we have

$$
\begin{aligned}
& \frac{\mathrm{d}}{\mathrm{d} x_{2}}=\frac{1}{\lambda_{h}} \frac{\mathrm{d}}{\mathrm{d} X_{2}}=\frac{1}{H \lambda_{h}} \frac{\mathrm{d}}{\mathrm{d} \bar{X}_{2}}, \\
& \frac{\mathrm{d}^{2}}{\mathrm{~d} x_{2}^{2}}=\frac{1}{H^{2} \lambda_{h}^{2}} \frac{\mathrm{d}^{2}}{\mathrm{~d} \bar{X}_{2}^{2}}-\frac{1}{H^{2} \lambda_{h}^{3}} \frac{\mathrm{d} \lambda_{h}}{\mathrm{~d} \bar{X}_{2}} \frac{\mathrm{d}}{\mathrm{d} \bar{X}_{2}},
\end{aligned}
$$

where $\bar{X}_{2}=X_{2} / H$. Therefore, Eqs. (14) and (15) can be re-written as

$$
\begin{aligned}
& \frac{\mathrm{d}^{2} U_{1}}{\mathrm{~d} \bar{X}_{2}^{2}}+\lambda_{h}^{-2}\left[f_{1}\left(\bar{X}_{2}\right)-\lambda_{h} \frac{\mathrm{d} \lambda_{h}}{\mathrm{~d} \bar{X}_{2}}\right] \frac{\mathrm{d} U_{1}}{\mathrm{~d} \bar{X}_{2}}-\bar{\omega}^{2}\left(1+\lambda_{h} \xi_{h}\right) U_{1} \\
& -\bar{\omega} \xi_{h} \frac{\mathrm{d} U_{2}}{\mathrm{~d} \bar{X}_{2}}-\bar{\omega} \lambda_{h}^{-1} f_{1}\left(\bar{X}_{2}\right) U_{2}=0, \\
& \bar{\omega} \xi_{h} \frac{\mathrm{d} U_{1}}{\mathrm{~d} \bar{X}_{2}}+\bar{\omega} \lambda_{h}^{-1} f_{2}\left(\bar{X}_{2}\right) U_{1}+\lambda_{h}^{-1}\left(\xi_{h}+\lambda_{h}\right) \frac{\mathrm{d}^{2} U_{2}}{\mathrm{~d} \bar{X}_{2}^{2}}
\end{aligned}
$$




$$
+\lambda_{h}^{-2}\left[f_{3}\left(\bar{X}_{2}\right)-\left(\xi_{h}+\lambda_{h}\right) \frac{\mathrm{d} \lambda_{h}}{\mathrm{~d} \bar{X}_{2}}\right] \frac{\mathrm{d} U_{2}}{\mathrm{~d} \bar{X}_{2}}-\bar{\omega}^{2} U_{2}=0,
$$

where $\quad \bar{\omega}=\omega H \quad, \quad f_{1}\left(\bar{X}_{2}\right)=\frac{\lambda_{h}}{N \Omega} \frac{\mathrm{d}}{\mathrm{d} \bar{X}_{2}}\left(\lambda_{h} N \Omega\right) \quad, \quad f_{2}\left(\bar{X}_{2}\right)=\frac{\lambda_{h}}{N \Omega} \frac{\mathrm{d}}{\mathrm{d} \bar{X}_{2}}\left[N \Omega\left(\xi_{h}-\lambda_{h}\right)\right]$, $f_{3}\left(\bar{X}_{2}\right)=\frac{\lambda_{h}}{N \Omega} \frac{\mathrm{d}}{\mathrm{d} \bar{X}_{2}}\left[N \Omega\left(\xi_{h}+\lambda_{h}\right)\right]$. Correspondingly, the boundary condition (18) becomes

$$
\frac{\mathrm{d} U_{1}}{\mathrm{~d} \bar{X}_{2}}-\bar{\omega} \lambda_{h} U_{2}=0 \text { and } \bar{\omega} \lambda_{h}\left(\xi_{h}-\lambda_{h}\right) U_{1}+\left(\xi_{h}+\lambda_{h}\right) \frac{\mathrm{d} U_{2}}{\mathrm{~d} \bar{X}_{2}}=0, \text { at } \bar{X}_{2}=1 .
$$

The number of nodes is taken as $m$ and the spacing between any two adjacent nodes is then $\Delta \bar{X}=1 /(m-1)$ in the reference configuration. The nodes may be numbered from 1 at the bottom surface of the layer $\left(\bar{X}_{2}=0\right)$ to $m$ at the top surface $\left(\bar{X}_{2}=1\right)$. By applying the central difference formulae to the node $k$ in the domain $(1<k<m)$, the normalized equilibrium equations (45) and (46) are discretized as

$$
\begin{aligned}
& A_{1} U_{1}^{(k-1)}+A_{2} U_{2}^{(k-1)}+A_{3} U_{1}^{(k)}+A_{4} U_{2}^{(k)}+A_{5} U_{1}^{(k+1)}+A_{6} U_{2}^{(k+1)}=0, \\
& B_{1} U_{1}^{(k-1)}+B_{2} U_{2}^{(k-1)}+B_{3} U_{1}^{(k)}+B_{4} U_{2}^{(k)}+B_{5} U_{1}^{(k+1)}+B_{6} U_{2}^{(k+1)}=0,
\end{aligned}
$$

where the superscript refers to the node number and the coefficients, $A_{i}\left(\omega H, \bar{X}_{2}^{(k)}\right)$ and $B_{i}\left(\omega H, \bar{X}_{2}^{(k)}\right)$, are evaluated at the node $k$ with $\bar{X}_{2}^{(k)}=(k-1) /(m-1)$ as

$$
\begin{aligned}
& A_{1}=(m-1)^{2}-\frac{1}{2}(m-1) \lambda_{h}^{-2}\left[f_{1}\left(\bar{X}_{2}\right)-\lambda_{h} \frac{\mathrm{d} \lambda_{h}}{\mathrm{~d} \bar{X}_{2}}\right], \\
& A_{2}=-A_{6}=-B_{1}=B_{5}=\frac{1}{2}(m-1) \bar{\omega} \xi_{h}, \\
& A_{3}=-2(m-1)^{2}-\bar{\omega}^{2}\left(1+\lambda_{h} \xi_{h}\right), \\
& A_{4}=-\bar{\omega} \lambda_{h}^{-1} f_{1}\left(\bar{X}_{2}\right), \\
& A_{5}=(m-1)^{2}+\frac{1}{2}(m-1) \lambda_{h}^{-2}\left[f_{1}\left(\bar{X}_{2}\right)-\lambda_{h} \frac{\mathrm{d} \lambda_{h}}{\mathrm{~d} \bar{X}_{2}}\right], \\
& B_{2}=(m-1)^{2} \lambda_{h}^{-1}\left(\xi_{h}+\lambda_{h}\right)-\frac{1}{2}(m-1) \lambda_{h}^{-2}\left[f_{3}\left(\bar{X}_{2}\right)-\left(\xi_{h}+\lambda_{h}\right) \frac{\mathrm{d} \lambda_{h}}{\mathrm{~d} \bar{X}_{2}}\right], \\
& B_{3}=\bar{\omega} \lambda_{h}^{-1} f_{2}\left(\bar{X}_{2}\right), \\
& B_{4}=-\bar{\omega}^{2}-2(m-1)^{2} \lambda_{h}^{-1}\left(\xi_{h}+\lambda_{h}\right),
\end{aligned}
$$


$B_{6}=(m-1)^{2} \lambda_{h}^{-1}\left(\xi_{h}+\lambda_{h}\right)+\frac{1}{2}(m-1) \lambda_{h}^{-2}\left[f_{3}\left(\bar{X}_{2}\right)-\left(\xi_{h}+\lambda_{h}\right) \frac{\mathrm{d} \lambda_{h}}{\mathrm{~d} \bar{X}_{2}}\right]$.

It should be noted that the coefficients $A_{i}$ and $B_{i} \quad(i=1,2, \cdots, 6)$ include differentiations of $\lambda_{h}$ and $\xi_{h}$ with respect to $\bar{X}_{2}$ at each node. For given functions $N\left(X_{2}\right)$ and $\chi\left(X_{2}\right), \lambda_{h}$ at each node is obtained by solving Eq. (1) and the corresponding $\xi_{h}$ is calculated accordingly. Differentiations of $\lambda_{h}$ and $\xi_{h}$ are then calculated by central difference.

At the bottom surface of the layer $\left(\bar{X}_{2}=0\right)$, the boundary condition (16) requires that

$$
U_{1}^{(1)}=U_{2}^{(1)}=0,
$$

which is used directly in Eqs. (48) and (49) for $k=2$.

At the top surface of the layer $\left(\bar{X}_{2}=1\right.$ ), Eqs. (48) and (49) for $k=m$ include displacements at a fictitious node $k=m+1$. By discretizing the boundary condition (18) with central difference, we obtain

$$
\begin{aligned}
& U_{1}^{(m+1)}=U_{1}^{(m-1)}+C_{1} U_{2}^{(m)}, \\
& U_{2}^{(m+1)}=U_{2}^{(m-1)}+C_{2} U_{1}^{(m)},
\end{aligned}
$$

where $C_{1}=2(m-1)^{-1} \bar{\omega} \lambda_{h}$ and $C_{2}=-2(m-1)^{-1} \bar{\omega} \lambda_{h} \frac{\xi_{h}-\lambda_{h}}{\xi_{h}+\lambda_{h}}$. Substituting Eqs. (51) and (52) into Eqs. (48) and (49) for $k=m$, we obtain that

$$
\begin{aligned}
& \left(A_{1}+A_{5}\right) U_{1}^{(m-1)}+\left(A_{2}+A_{6}\right) U_{2}^{(m-1)}+\left(A_{3}+A_{6} C_{2}\right) U_{1}^{(m)}+\left(A_{4}+A_{5} C_{1}\right) U_{2}^{(m)}=0, \\
& \left(B_{1}+B_{5}\right) U_{1}^{(m-1)}+\left(B_{2}+B_{6}\right) U_{2}^{(m-1)}+\left(B_{3}+B_{6} C_{2}\right) U_{1}^{(m)}+\left(B_{4}+B_{5} C_{1}\right) U_{2}^{(m)}=0 .
\end{aligned}
$$

Therefore, by Eqs. (48) and (49) with $k=2,3, \ldots, m-1$ as well as Eqs. (53) and (54), a set of algebraic equations are obtained in a matrix form as

$$
\mathbf{M U}=\mathbf{0} \text {, }
$$

where $\mathbf{M}$ is a $2(m-1) \times 2(m-1)$ matrix, and $\mathbf{U}=\left[U_{1}^{(2)}, U_{2}^{(2)}, \cdots, U_{1}^{(m)}, U_{2}^{(m)}\right]^{\mathrm{T}}$. The eigenvalue problem in Section 2.2 is thus discretized, and a nontrivial solution requires that the determinant of the coefficient matrix vanish, namely

$$
\operatorname{det} \mathbf{M}=f_{m}\left(\bar{\omega}, \mu_{\mathrm{c}}\right)=0 .
$$

Similar to Eq. (40) by the state space method, Eq. (56) presents an implicit relation between the critical chemical potential $\mu_{\mathrm{c}}$ and the dimensionless wave number $\bar{\omega}$, which depends on the discretized material properties $N^{(k)} \Omega$ and $\chi^{(k)}$. The critical swelling ratio 
at each node $\lambda_{\mathrm{c}}^{(k)}$ is then computed from Eq. (1) with $\mu=\mu_{\mathrm{c}}$. The average critical swelling ratio for the graded hydrogel layer is obtained approximately as

$$
\lambda_{\mathrm{c}}=\frac{1}{m-1} \sum_{k=1}^{m} \lambda_{\mathrm{c}}^{(k)}-\frac{\lambda_{\mathrm{c}}^{(1)}+\lambda_{\mathrm{c}}^{(m)}}{2(m-1)} .
$$

By this method, a large number of nodes are often used in order to achieve convergence, and we employ a bisection method for solving the nonlinear equation (56).

\section{Results and discussion}

In this section, by using the state space method (Section 3) and the finite difference method (Section 4), we consider swell-induced surface instabilities for homogeneous hydrogel layers, hydrogel bilayers, and continuously graded hydrogel layers. For both methods, convergences of the approximate solutions are demonstrated by increasing the number of sub-layers or discrete nodes, in comparison with the analytical solutions for homogeneous layers (Kang and Huang, 2010a) and bilayers (Wu et al., 2013).

\subsection{Surface instability of a homogeneous hydrogel layer}

For a homogeneous hydrogel layer, the critical condition for onset of surface instability was predicted by an analytical method (Kang and Huang, 2010a). As shown in Fig. 3, for given material properties, the critical chemical potential and the corresponding critical swelling ratio depend on the normalized perturbation wave number. The minimum critical swelling ratio is obtained at the short wave limit $(\omega H \rightarrow \infty)$, as predicted by Eq. (33). By the state space method, only one sublayer $(n=1)$ is necessary in this case and the result is identical to the analytical solution. By the finite difference method, however, a large number of nodes are needed to achieve convergence. As shown in Fig. 4, the finite difference method converges more quickly for the long-wave modes than for the short-wave modes. To obtain the critical swelling ratio at the short-wave limit $(\omega H \rightarrow \infty), m=2000$ is found to be sufficient. Clearly, the state space method is more efficient than the finite difference method for the case of a homogeneous layer. 


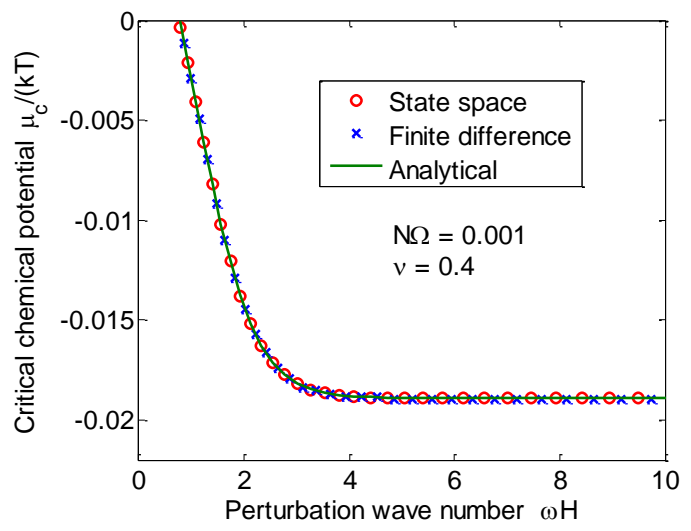

(a)

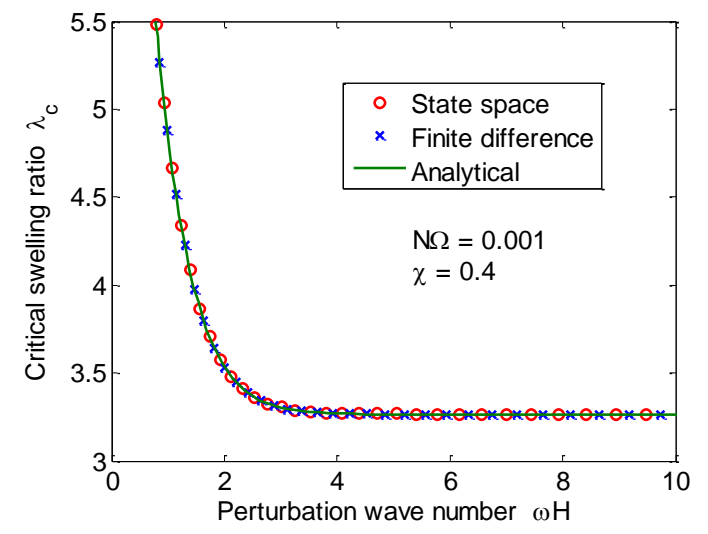

(b)

Fig. 3. (a) The critical chemical potential and (b) the corresponding swelling ratio for a homogeneous hydrogel layer, comparing the results by the state space method $(n=1)$ and the finite difference method ( $m=2000)$ with the analytical solutions by Kang and Huang (2010a).

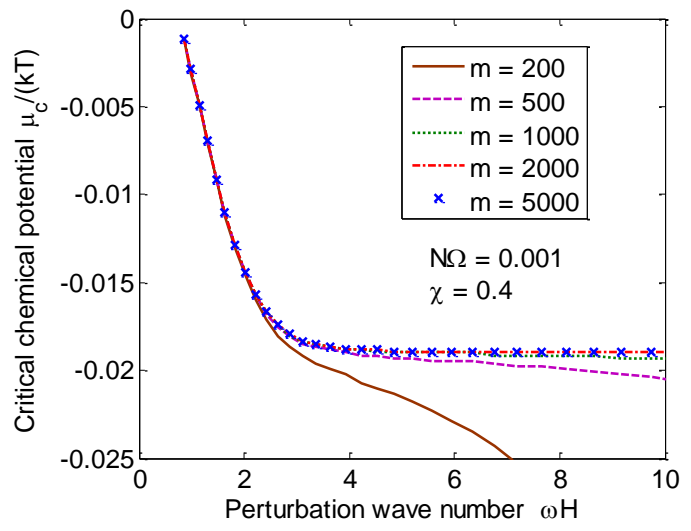

(a)

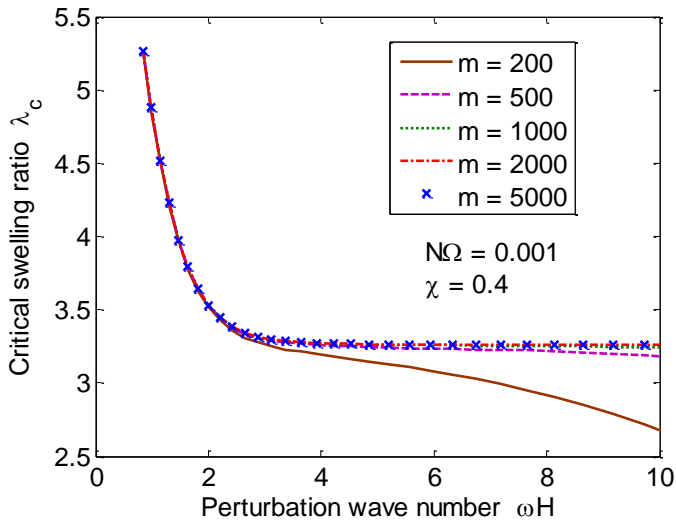

(b)

Fig. 4. Convergence of the finite difference method for a homogeneous hydrogel layer: (a) the critical chemical potential; (b) the corresponding swelling ratio.

\subsection{Surface instability of hydrogel bilayers}

For a hydrogel bilayer, the critical condition of surface instability depends on material properties in the two sub-layers (Wu et al., 2013). Since the material properties are discontinuous at the interface between the upper layer (film) and the underlayer (substrate), both $N \Omega$ and $\chi$ and their differentiations with respect to $\bar{X}_{2}$ are uncertain at the interface, and thus the finite difference method is unsuitable in this case. Approximate solutions may be obtained by the finite difference method by replacing the sharp interface with a smooth transition, which however would require a very large number of nodes to converge to the 
bilayer solution. On the other hand, by the state space method, only two sub-layers $(n=2)$ are needed, and the results are identical to the previous analytical solution (Wu et al., 2013), as shown in Fig. 5. Two types of bilayers are considered. For the soft-on-hard bilayer, the underlayer is stiffer than the upper layer $\left(N_{1}>N_{2}\right)$, and the minimum critical swelling ratio is obtained at the short wave limit ( $\omega H \rightarrow \infty$ ), similar to the homogeneous case. In such a case, numerical simulation in the previous study (Wu et al., 2013) showed creasing of the upper surface, possibly because surface wrinkling of any finite wavelength would be unstable and tend to collapse into localized creases. For the hard-on-soft bilayer $\left(N_{1}<N_{2}\right)$, the underlayer is softer and the minimum swelling ratio corresponds to a long-wave wrinkling mode $(\omega H \approx 6)$. In such a case, numerical simulation (Wu et al., 2013) showed wrinkling of the upper layer at the onset of surface instability. Clearly the state space method is quite effective for the case of hydrogel bilayers, and it can be readily extended to multilayer hydrogels.

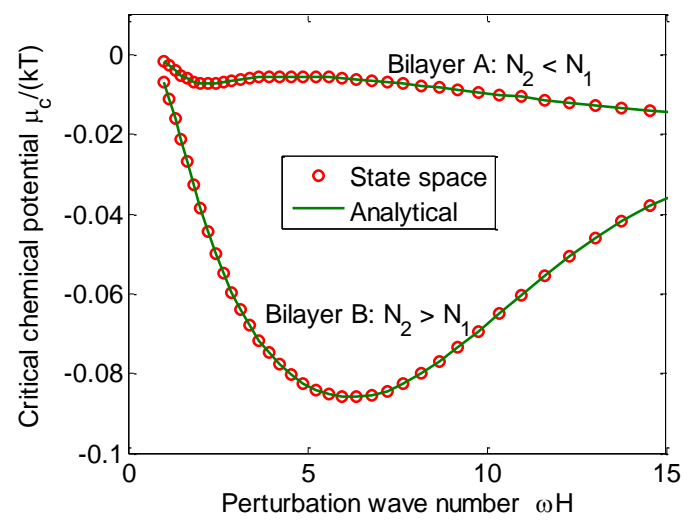

(a)

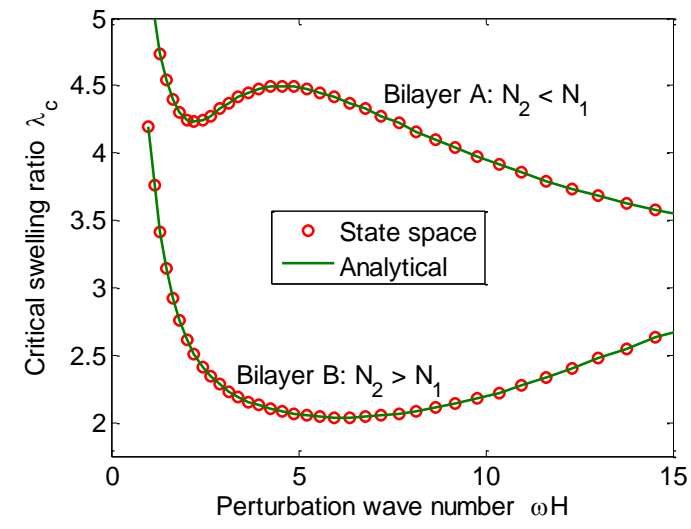

(b)

Fig. 5. (a) The critical chemical potential and (b) the corresponding swelling ratio versus the perturbation wave number for two types of hydrogel bilayers (A: $N_{2} \Omega=5 \times 10^{-4}$; B: $N_{2} \Omega=2 \times 10^{-3}$ ), both with $N_{1} \Omega=10^{-3}$ for the underlayer, $\chi_{1}=\chi_{2}=0.4$, and $H_{1} / H_{2}=9$ for the thickness ratio.

\subsection{Hydrogel layers with continuously graded material properties}

To predict onset of surface instability for hydrogel layers with continuously graded material properties, we consider two types of graded hydrogel layers: one with linearly graded and the other with exponentially graded crosslink density in the thickness direction. Since the effective number of polymer chains per unit volume is proportional to the crosslink density, we have

$$
N\left(\bar{X}_{2}\right)=N_{\mathrm{b}}+\left(N_{\mathrm{t}}-N_{\mathrm{b}}\right) \bar{X}_{2},
$$


or

$$
N\left(\bar{X}_{2}\right)=N_{\mathrm{b}}+\left(N_{\mathrm{t}}-N_{\mathrm{b}}\right) \frac{\mathrm{e}^{\eta \bar{X}_{2}}-1}{\mathrm{e}^{\eta}-1}
$$

where $N_{\mathrm{b}}=N\left(\bar{X}_{2}=0\right), \quad N_{\mathrm{t}}=N\left(\bar{X}_{2}=1\right)$, and the parameter $\eta$ is a shape factor for the exponential function. When $\eta=0$, the exponential function in Eq. (59) reduces to the linear function in Eq. (58). The Flory parameter is assumed to be a constant, $\chi=0.4$, in both cases. Figure 6 presents the dimensionless material property $N \Omega$ varying from the bottom surface with $N_{\mathrm{b}} \Omega=0.001$ to the top surface with $N_{\mathrm{t}} \Omega=0.01$ for $\eta=-5,0,5$, and 10 .

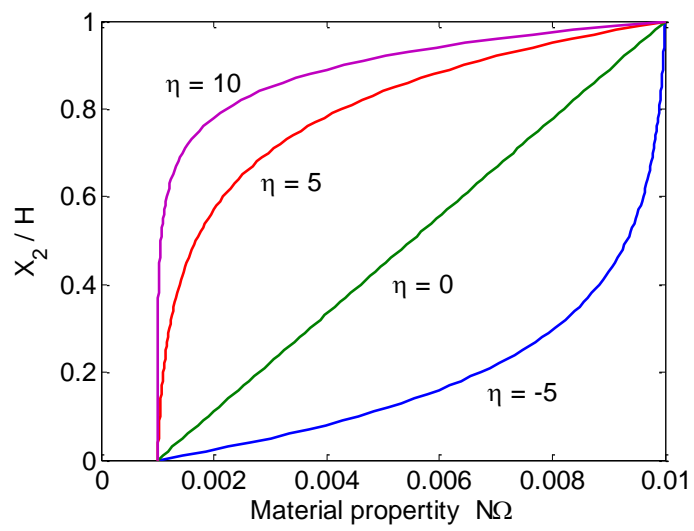

Fig. 6. The linear and exponential variations of the material property $(N \Omega)$ in the thickness direction of a hydrogel layer with $N_{\mathrm{b}} \Omega=0.001$ and $N_{\mathrm{t}} \Omega=0.01$.

Figure 7 shows the critical condition for surface instability of a linearly graded hydrogel layer with $N_{\mathrm{b}} \Omega=0.001$ and $N_{\mathrm{t}} \Omega=0.01$. By the state space method, the layer is divided into $n=10,20,50,100$ uniform sub-layers. The critical chemical potential $\mu_{\mathrm{c}}$ and the corresponding swelling ratio $\lambda_{\mathrm{c}}$ are obtained as functions of perturbation wave number $\omega H$. The state space solution converges fairly quickly with the increasing number of sub-layers $n$. The results for $n=50$ and $n=100$ are indistinguishable. Similar to the case of a hard-on-soft bilayer (Fig. 5), the minimum swelling ratio corresponds to a long-wave wrinkling mode ( $\omega H \approx 2$ ), and thus wrinkling is expected at the onset of surface instability. The finite difference method can also be used in this case. For convergence, the linearly graded layer is discretized into 2000 nodes as for the homogeneous layer. As shown in Fig. 7, the results obtained by both methods are in excellent agreement. To our knowledge, no analytical solution or other numerical results can be found in the literature for the continuously graded hydrogel layers. 


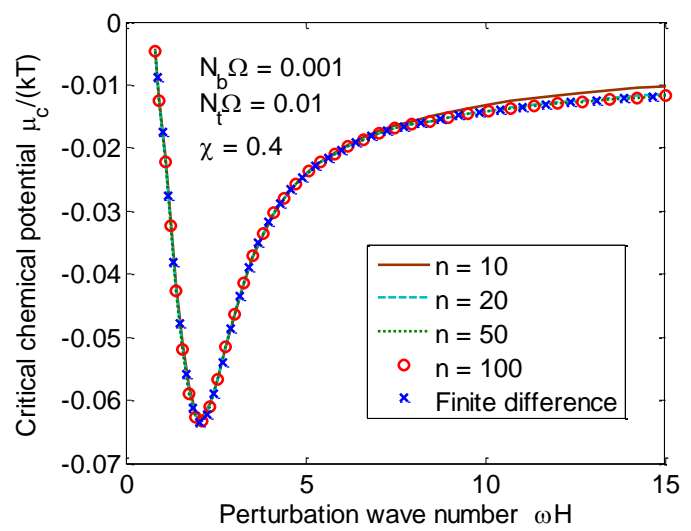

(a)

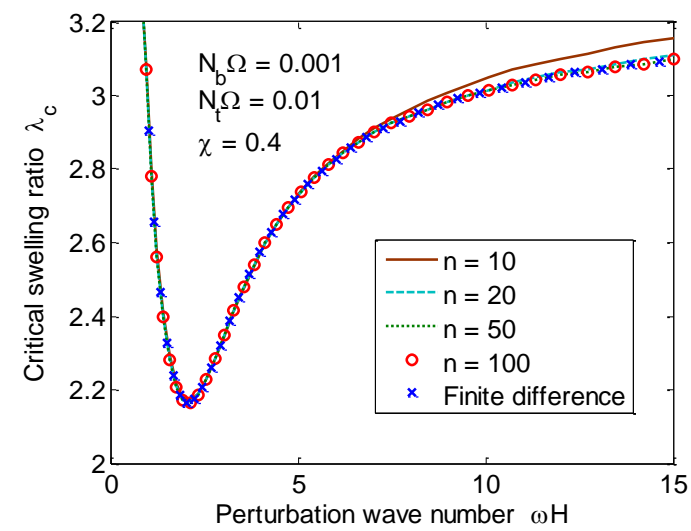

(b)

Fig. 7. Convergence of the state space method for a linearly graded hydrogel layer: (a) The critical chemical potential and (b) the corresponding swelling ratio, in comparison with the finite difference results denoted by " $x$ ".

Figure 8 shows the critical chemical potential and the corresponding swelling ratio for three linearly graded hydrogel layers with different dimensionless material property $N_{\mathrm{t}} \Omega$ and the same $N_{\mathrm{b}} \Omega$. As $N_{\mathrm{t}} \Omega$ increases, the swelling ratio decreases, and the corresponding wave number at the minimum critical swelling ratio decreases as well. Again, the results obtained by the state space method $(n=50)$ and the finite difference method $(m=2000)$ are in close agreement.

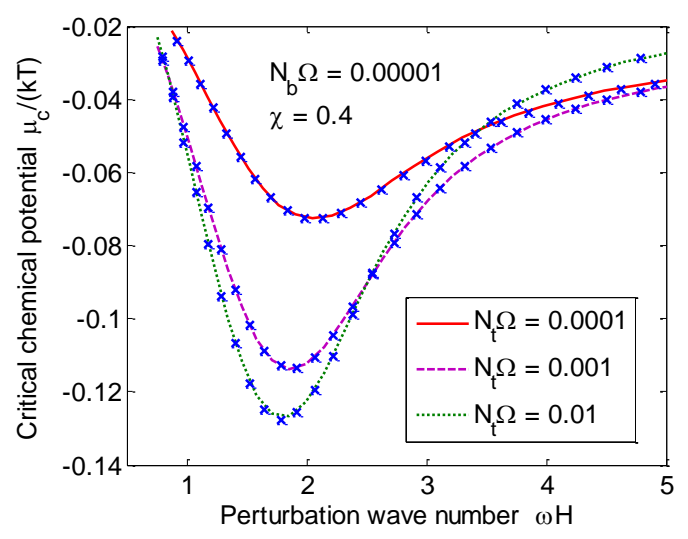

(a)

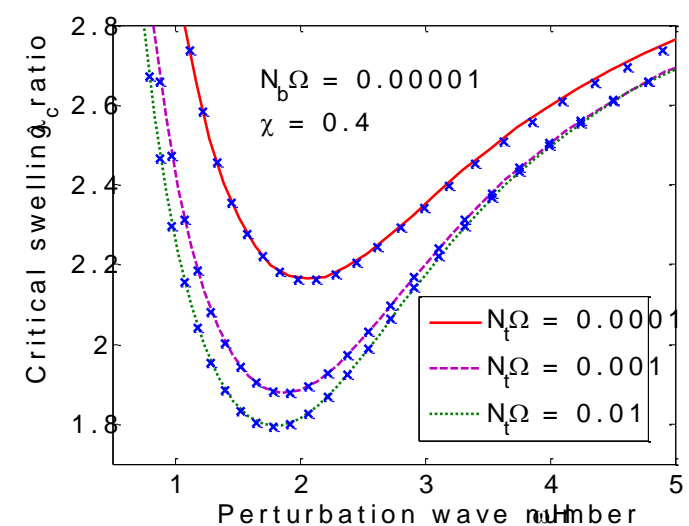

(b)

Fig. 8. (a) The critical chemical potential and (b) the corresponding swelling ratio versus the perturbation wave number for a linearly graded hydrogel layer obtained by the state space method, in comparison with the finite difference results denoted by " $x$ ". 
Similar results are obtained for the exponentially graded hydrogel layers. The minimum critical swelling ratio $\lambda_{\mathrm{c}}^{*}$ and the corresponding wavelength $L^{*}=2 \pi / \omega^{*}$, normalized by the thickness $H$, are plotted in Fig. 9 as functions of the shape factor $\eta$. The results by both the state space method and the finite difference method are in excellent agreement. Remarkably, with the same values of $N_{\mathrm{b}} \Omega$ and $N_{\mathrm{t}} \Omega$, the critical swelling ratio and the corresponding wavelength depend on grading profile via the shape factor $\eta$. As $\eta$ increases, the critical swelling ratio $\lambda_{\mathrm{c}}^{*}$ decreases monotonically, which can be considerably lower than that predicted for a homogeneous layer in the range of 2.5 3.4 (Kang and Huang, 2010a). On the other hand, the corresponding critical wavelength depends on the shape factor $\eta$ non-monotonically (Fig. 9b). This result suggests that the critical condition and the surface instability pattern depend on how the material properties vary in the hydrogel layers.

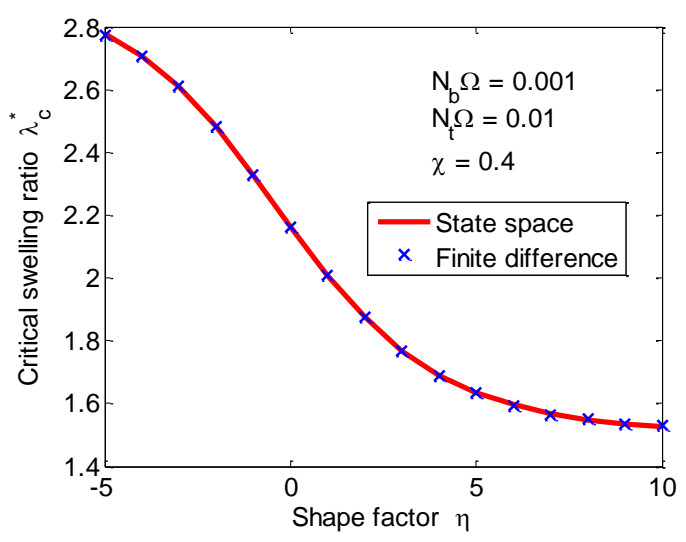

(a)

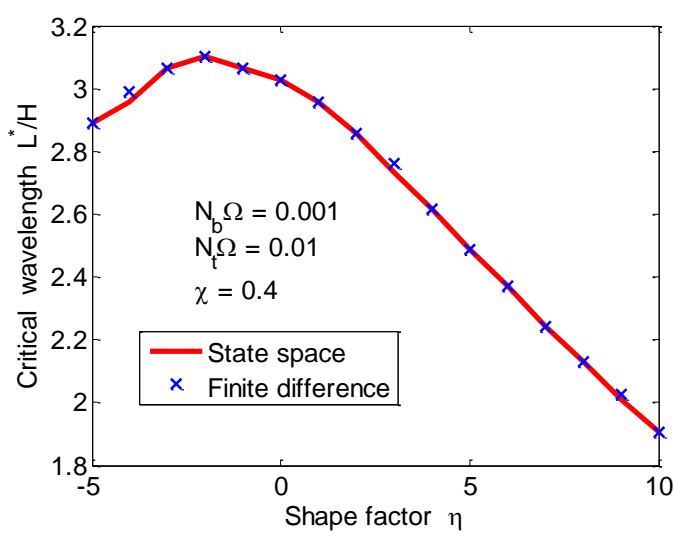

(b)

Fig. 9. (a) The critical swelling ratio and (b) the corresponding wavelength for exponentially graded hydrogel layers versus the shape factor $\eta$ ranging from -5 to 10 .

Figure 10 plots the critical swelling ratio $\lambda_{\mathrm{c}}^{*}$ and the corresponding normalized wavelength $L^{*} / H$ for the exponentially graded hydrogel layers with $N_{\mathrm{t}} \Omega$ ranging from $2 \times 10^{-5}$ to 0.1 whereas $N_{\mathrm{b}} \Omega=10^{-5}$ and $\eta=5$. As $N_{\mathrm{t}} \Omega$ increases, the minimum critical swelling ratio $\lambda_{\mathrm{c}}^{*}$ decreases. Meanwhile, the corresponding normalized wavelength $L^{*} / H$ increases monotonically with the increasing $N_{\mathrm{t}} \Omega$ as shown in Fig. 10b. Therefore, by controlling the crosslink density near the top surface and the depth-wise variation in the hydrogel layer, different critical wavelength of swell-induced surface instability can be obtained. 


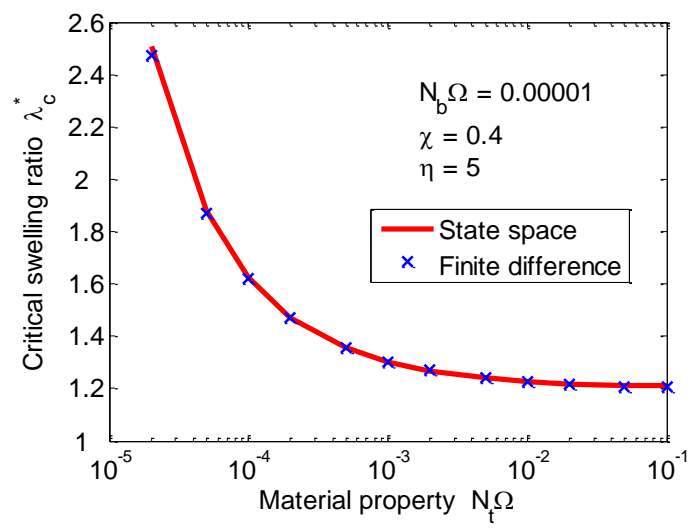

(a)

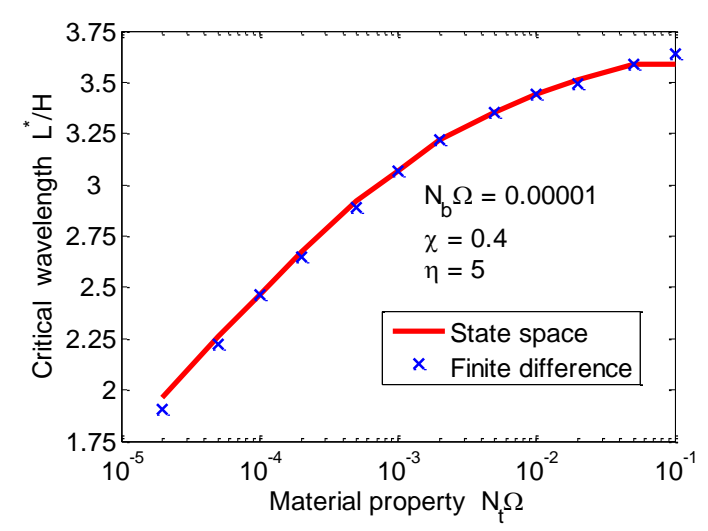

(b)

Fig. 10. (a) The critical swelling ratio and (b) the corresponding wavelength for exponentially graded hydrogel layers with $N_{\mathrm{t}} \Omega$ ranging from $2 \times 10^{-5}$ to $10^{-1}$.

\subsection{Surface instability of graded hydrogel bilayers}

To compare with the experiments in Guvendiren et al. (2009), we consider a bilayer model with a graded top layer on a uniform bottom layer. The top layer is assumed to have a linearly graded material property, namely

$$
N\left(X_{2}\right)= \begin{cases}N_{\mathrm{b}}+\left(N_{\mathrm{t}}-N_{\mathrm{b}}\right) \frac{X_{2}-H_{\mathrm{b}}}{H-H_{\mathrm{b}}}, & H_{\mathrm{b}}<X_{2}<H \\ N_{\mathrm{b}}, & 0<X_{2}<H_{\mathrm{b}}\end{cases}
$$

where $N_{\mathrm{b}}$ and $N_{\mathrm{t}}$ refer to the effective numbers of polymer chains per unit volume at the bottom and top surfaces, respectively, $H_{\mathrm{b}}$ is the thickness of the bottom layer. The Flory parameter $\chi$ is assumed to be the same in both layers. By the state space method, the graded top layer is divided into 50 sub-layers, while a single sublayer is used for the bottom layer. The predicted critical swelling ratio and the corresponding wavelength are presented in Fig. 11, in which $N_{\mathrm{t}} \Omega$ is varied, but $N_{\mathrm{b}} \Omega=0.001$ and $H_{\mathrm{b}} / H=2 / 3$ are fixed. The critical swelling ratio $\lambda_{\mathrm{c}}^{*}$ decreases with increasing $N_{\mathrm{t}} \Omega$, while the corresponding wavelength $L^{*} / H$ increases. In particular, the critical swelling ratio drops below 1.2 for $N_{\mathrm{t}} \Omega>0.05$, comparable to the critical value of $\sim 1.12$ as observed in experiments (Guvendiren et al. 2010a). However, Guvendiren et al. (2009) suggested that the top surface has a lower crosslink density in their experiments with the PHEMA films due to diffused oxygen inhibiting photopolymerization and crosslinking of the polymer. If that was the case, based on the present stability analysis, the critical swelling ratio would be much larger and the critical 
wavelength would be much shorter (approaching zero), similar to the soft-on-hard bilayer case as shown in Fig. 5. As a result, instead of the wrinkles as observed in their experiments, creases would be expected. Therefore, the prediction of the present stability analysis is consistent with the experiments only if the top surface actually had a higher crosslink density.

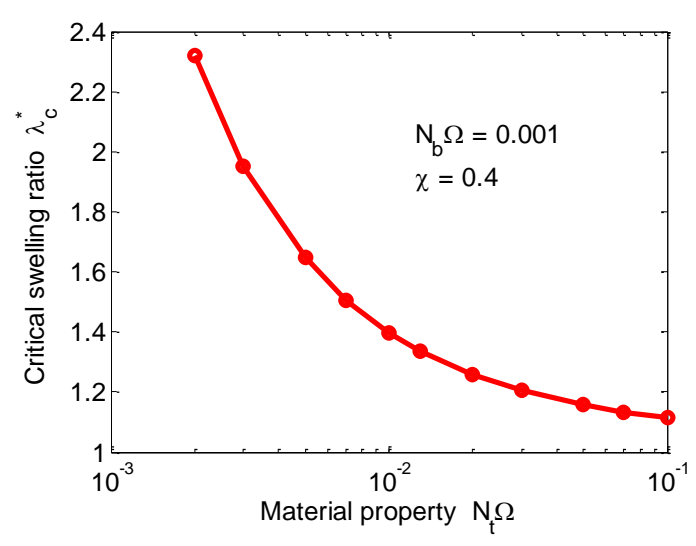

(a)

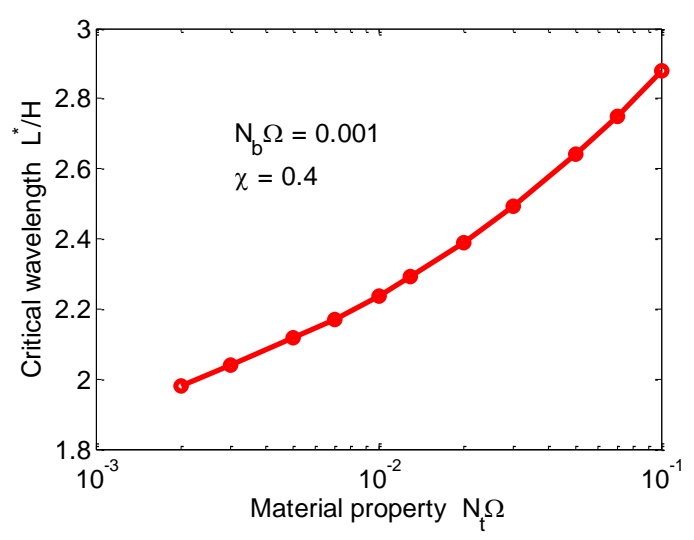

(b)

Fig. 11. (a) The critical swelling ratio and (b) the corresponding wavelength for a linearly graded top layer on a uniform bottom layer.

\section{Summary}

In this paper, a state space method and a finite difference method were developed for predicting the onset of surface instability in hydrogel layers with material properties varying in the thickness direction. Both methods were verified by comparing to the analytical solutions of homogeneous hydrogel layers. The results from the state space method are also in complete agreement with the corresponding analytical solution for hydrogel bilayers. While the finite difference method often requires a large number of nodes to achieve convergence, the state space method is most efficient for multilayers with discontinuous variations in material properties and requires relatively fewer sub-layers for continuously graded layers. The results for linearly and exponentially graded hydrogel layers show that the critical swelling ratio and corresponding critical wavelength depend on the gradient profile of the crosslink density. The present study may provide theoretical guidance for analyzing and designing surface instability and surface patterns in hydrogel layers.

\section{Acknowledgements}

ZW and YL gratefully acknowledge funding of this work by Nature Science Foundation 
of China through Grant No. 11572108. NB and RH gratefully acknowledge funding by National Science Foundation of USA through Grant No. 1538658. 


\section{References}

Beebe, D.J., Moore, J.S., Bauer, J.M., Yu, Q., Liu, R.H., Devadoss, C., Jo, B.H., 2000. Functional hydrogel structures for autonomous flow control inside microfluidic channels. Nature 404, 588-590.

Cao, Y.-P., Jia, F., Zhao, Y., Feng, X.-Q., Yu, S.-W., 2012. Buckling and post-buckling of a stiff film resting on an elastic graded substrate. Int. J. Solids Struct. 49, 1656-1664.

Derusso, P.M., Roy, R.J., Close, C.M., Desrochers, A.A., 1998. State Variables for Engineers. John Wiley \& Sons, Inc., New York.

Diab, M., Kim, K.S., 2014. Ruga-formation instabilities of a graded stiffness boundary layer in a neo-hookean solid. Proc. R. Soc. A. 470, 218-218.

Elgerd, O.L., 1967. Control System Theory. McGraw-Hill, Kogakusha, Inc., Tokyo.

Guvendiren, M., Burdick, J.A., Yang S., 2010a. Kinetic study of swelling-induced surface pattern formation and ordering in hydrogel films with depth-wise crosslinking gradients. Soft Matter 6, 2044-2049.

Guvendiren, M., Burdick, J.A., Yang S., 2010b. Solvent induced transition from wrinkles to creases in thin film gels with depth-wise crosslinking gradients. Soft Matter 6, 5795-5801.

Guvendiren, M., Yang, S., Burdick, J.A., 2009. Swelling-induced surface patterns in hydrogels with gradient crosslinking density. Adv. Funct. Mater. 19, 3038-3045.

Harrison, C., Stafford, C.M., Zhang, W., Karim, A., 2004. Sinusoidal phase grating created by a tunably buckled surface. Appl. Phys. Lett. 85, 4016-4018.

Kang, M.K., Huang, R., 2010a. Swell-induced surface instability of confined hydrogel layers on substrates. J. Mech. Phys. Solids 58, 1582-1598.

Kang, M.K., Huang, R., 2010b. Effect of surface tension on swell-induced surface instability of substrate-confined hydrogel layers. Soft Matter 6, 5736-5742.

Lee, D., Triantafyllidis, N., Barber, J.R., Thouless, M.D., 2008. Surface instability of an elastic half space with material properties varying with depth. J. Mech. Phys. Solids 56, $858-868$.

Schaffer, E., Thurn-Albrecht, T., Russell, T.P., Steiner, U., 2000. Electrically induced structure formation and pattern transfer. Nature 403, 874-877.

Stafford, C.M., Harrison, C., Beers, K.L., Karim, A., Amis, E.J., Vanlandingham, M.R., Kim, H.-C., Volksen, W., Miller R.D., Simonyi, E.E., 2004. A buckling-based metrology for measuring the elastic moduli of polymeric thin films. Nat. Mater. 3, 545-550.

Sugiura, S., Sumaru, K., Ohi, K., Hiroki, K., Takagi, T., Kanamori, T., 2007. Photoresponsive 
polymer gel microvalves controlled by local light irradiation. Sensor Actuat. A 140, $176-184$.

Sultan, E., Boudaoud, A., 2008. The buckling of a swollen thin gel layer bound to a compliant substrate. J. Appl. Mech. 75, 051002.

Tanaka, H., Tomita, H., Takasu, A., Hayashi, T., Nishi, T., 1992. Morphological and kinetic evolution of surface patterns in gels during the swelling process: evidence of dynamic pattern ordering. Phys. Rev. Lett. 68, 2794-2797.

Tanaka, T., Sun, S.-T., Hirokawa, Y., Katayama, S., Kucera, J., Hirose, Y., Amiya, T., 1987. Mechanical instability of gels at the phase transition. Nature 325, 796-798.

Toh, W., Ding, Z.W., Ng, T.Y., Liu, Z.S., 2015. Wrinkling of a polymeric gel during transient swelling. J. Appl. Mech. 82, 061004.

Tokarev, I., Minko, S., 2009. Stimuli-responsive hydrogel thin films. Soft Matter 5, 511-524.

Trujillo, V., Kim, J., Hayward, R.C., 2008. Creasing instability of surface-attached hydrogels. Soft Matter 4, 564-569.

Weiss, F., Cai, S., Hu, Y., Kang, M.K., Huang, R., Suo, Z., 2013. Creases and wrinkles on the surface of a swollen gel. J. Appl. Phys. 114, 073507.

Wu, Z., Bouklas, N., Huang, R., 2013. Swell-induced surface instability of hydrogel layers with material properties varying in thickness direction. Int. J. Solids Struct. 50, 578-587.

Wu, Z., Meng, J., Liu, Y., Li, H., Huang, R., 2014. A state space method for surface instability of elastic layers with material properties varying in thickness direction. J. Appl. Mech. 81,081003 . 